\title{
Technè
}

La science au service de l'histoire de l'art et de la préservation des biens culturels

48 | 2019

Les couleurs de l'Antique

\section{La couleur des bronzes antiques : recherches récentes}

The colour of ancient bronzes: recent research

\section{Sophie Descamps-Lequime}

\section{OpenEdition}

Journals

Édition électronique

URL : https://journals.openedition.org/techne/2323

DOI : 10.4000/techne.2323

ISSN : 2534-5168

Éditeur

C2RMF

Édition imprimée

Date de publication : 31 décembre 2019

Pagination : 72-82

ISBN : 978-2-11-152831-4

ISSN : $1254-7867$

Référence électronique

Sophie Descamps-Lequime, "La couleur des bronzes antiques : recherches récentes », Technè [En ligne], 48 | 2019, mis en ligne le 01 juillet 2021, consulté le 03 avril 2022. URL : http:// journals.openedition.org/techne/2323 ; DOI : https://doi.org/10.4000/techne.2323

La revue Technè. La science au service de l'histoire de l'art et de la préservation des biens culturels est mise à disposition selon les termes de la Licence Creative Commons Attribution - Pas d'Utilisation Commerciale - Pas de Modification 4.0 International. 


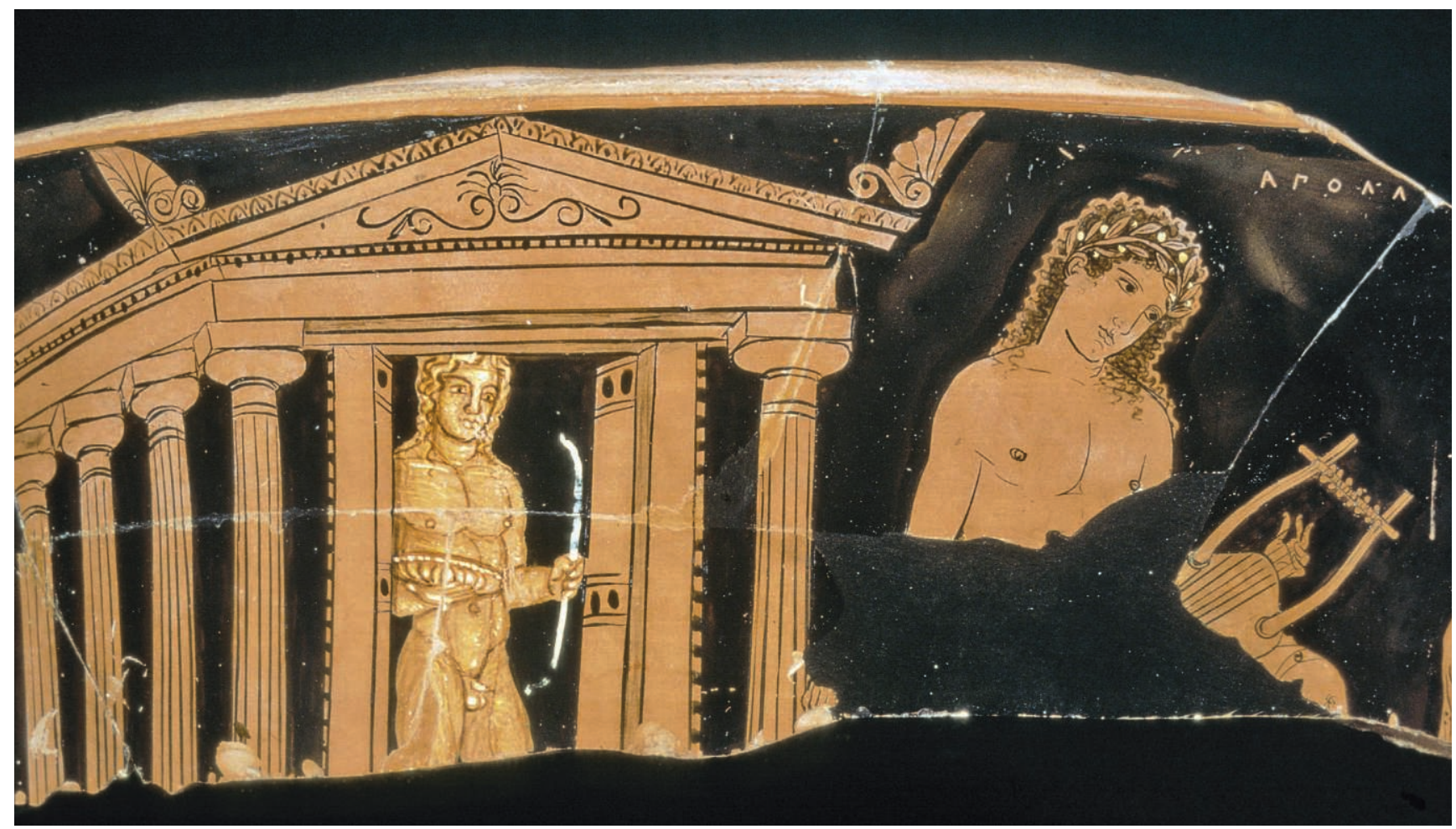

Fig. 1. Peintre de la naissance de Dionysos, fragment de cratère en calice, Tarente, premier quart du iv siècle av. J.-C., Amsterdam, Allard Pierson Museum (inv. 2579). @ Allard Pierson - The Collections of the University of Amsterdam. 


\section{La couleur des bronzes antiques : recherches récentes}

The colour of ancient bronzes: recent research

Résumé. Les effets de polychromie recherchés par les bronziers antiques reposaient essentiellement sur des techniques propres au travail du métal et non sur les pratiques picturales adoptées à la même époque par les sculpteurs sur pierre et par les coroplathes. Leur palette, liée à des incrustations et à des placages de métaux purs ou alliés, était donc limitée, même si l'une des sources de couleurs supplémentaires venait des yeux rapportés des statues. Les Anciens recherchaient l'éclat et la brillance du métal. Pour préserver la teinte métallique originelle des bronzes, proche d'un or plus ou moins rosé, et ralentir le processus d'altération du bronze, ils traitaient les épidermes à l'aide de substances bitumineuses ou de poix qui, paradoxalement, assombrissaient progressivement les statues. Cet assombrissement est probablement à l'origine d'un changement de goût, qui expliquerait peut-être l'apparition, avant la fin de l'époque hellénistique, de patines artificielles intentionnellement sombres, dont certaines ont été étudiées au $C 2 R M F$.

Mots-clés. Grands bronzes, bronzes peints, incrustations métalliques, polychromie, yeux, patines intentionnelles, bronze de Corinthe.
Abstract. Polychrome effects sought by ancient bronzesmiths basically relied on metalwork techniques rather than on pictorial practices adopted by stone sculptors and clay figurine makers in the same period. Employing inlays and claddings of pure metals or alloys, their palette was therefore limited, even if one of the sources of extra colours came from the eyes added to the statues.

In Antiquity, the shine and brilliance of metal were highly valued. To preserve the original metallic hue of the bronzes, close to a more or less pink gold, and to slow down the deterioration process, bronze sculptors coated the surface with bituminous substances or pitch which, paradoxically, gradually darkened the statues. This sombre appearance probably gave rise to a change in taste which would perhaps explain the emergence, before the end of the Hellenistic period, of artificial patinas that were deliberately dark, some of which have been studied at C2RMF.

Keywords. Large bronzes, painted bronzes, metallic inlays, polychromy, eyes, intentional patinas, Corinthian bronze.

\section{Un dossier compliqué par la rareté et l'altération des statues antiques en bronze}

Les grands bronzes grecs et romains aujourd'hui conservés sont extrêmement rares alors qu'ils se comptaient par milliers dans l'Antiquité. Leur disparition s'explique par un fait matériel. Il était possible de les refondre, une opération survenue quelques années seulement ou plusieurs siècles après leur création, afin de recycler les métaux qui les composaient. Les quelques œuvres parvenues jusqu'à nous ont échappé à ce destin contraire car elles avaient disparu fortuitement bien avant la fin de l'Empire romain, à cause de séismes, d'incendies ou du naufrage des bateaux qui les transportaient. Quelquesunes, trouvées trop tôt, n'ont pu bénéficier d'observations ou d'analyses précises et ont subi parfois des interventions irréversibles : des nettoyages excessifs empêchent souvent de retrouver leur surface d'abandon, de statuer sur les traitements qui leur ont été appliqués avant leur ensevelissement et sur l'existence ou non de patines intentionnelles antiques.

Cependant, les indices se multiplient heureusement désormais grâce à l'étude de trouvailles plus récentes, examinées dans le cadre d'une restauration et d'une collaboration étroite entre historiens d'art, archéologues, physicochimistes et restaurateurs.

L'étude en laboratoire s'avère ici déterminante pour fonder sur des bases scientifiques solides la compréhension des techniques antiques - et, partant, toute forme de restitution. Lorsqu'il s'agit de reproduire l'apparence originelle des statues de bronze antiques et leur polychromie, les tentatives,

Sophie Descamps-Lequime, conservateur général du patrimoine, musée du Louvre, département des Antiquités grecques, étrusques et romaines (sophie.descamps@louvre.fr). 


\section{Une collaboration scientifique entre le Louvre et le C2RMF : le cas des bronzes grecs et romains}

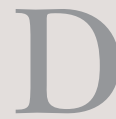

EPUIS une quinzaine d'années, le Centre de Recherche et de Restauration des Musées de France et le département des Antiquités grecques, étrusques et romaines du musée du Louvre mènent, en étroite collaboration, des recherches sur les procédés de fabrication (techniques de fonte à la cire perdue et soudage) et sur la polychromie originelle de la statuaire grecque et romaine en bronze.

Pilotée par Sophie Descamps (musée du Louvre) et Benoît Mille (C2RMF), l'enquête interdisciplinaire sur les techniques de fabrication, alliant données archéologiques, stylistiques et archéométriques (imagerie et analyses scientifiques de pointe), a bénéficié des contributions de chercheurs en physico-chimie des matériaux et en science de la conservation-restauration (Aurélia Azéma, Isabelle Biron, Alexis Blettner, Thierry Borel, Anne Bouquillon, Thomas Calligaro, Yvan Coquinot, Elsa Lambert, Juliette Langlois, Nathalie Pingaud, Romain Renaud et Dominique Robcis). Une enquête comparable associant sciences humaines (Sophie Descamps) et sciences de laboratoire (Marc Aucouturier, François Mathis et Dominique Robcis) a été conduite parallèlement sur les surfaces métalliques (dorure, patines intentionnelles antiques).

La synthèse des résultats enrichit en continu la base de données Héphaïstos, qui a pour objectif de dresser un large panorama de la variation spatio-temporelle des techniques de création des sculptures en bronze.

Les nombreux acquis de la recherche ont permis, dès 2005, de renouveler entièrement la lecture de certaines œuvres, tels l'Apollon Chatsworth ou l'encrier de Vaisonla-Romaine. Ils ont nourri plusieurs communications et publications communes. On mentionnera en particulier les actes, parus dans la revue Technè ( $\left.\mathrm{n}^{\circ} 45,2017\right)$, d'une journée d'étude sur les notions de surmoulages antiques et de datation des grands bronzes, organisée conjointement en 2013 à l'occasion de la présence au Louvre de

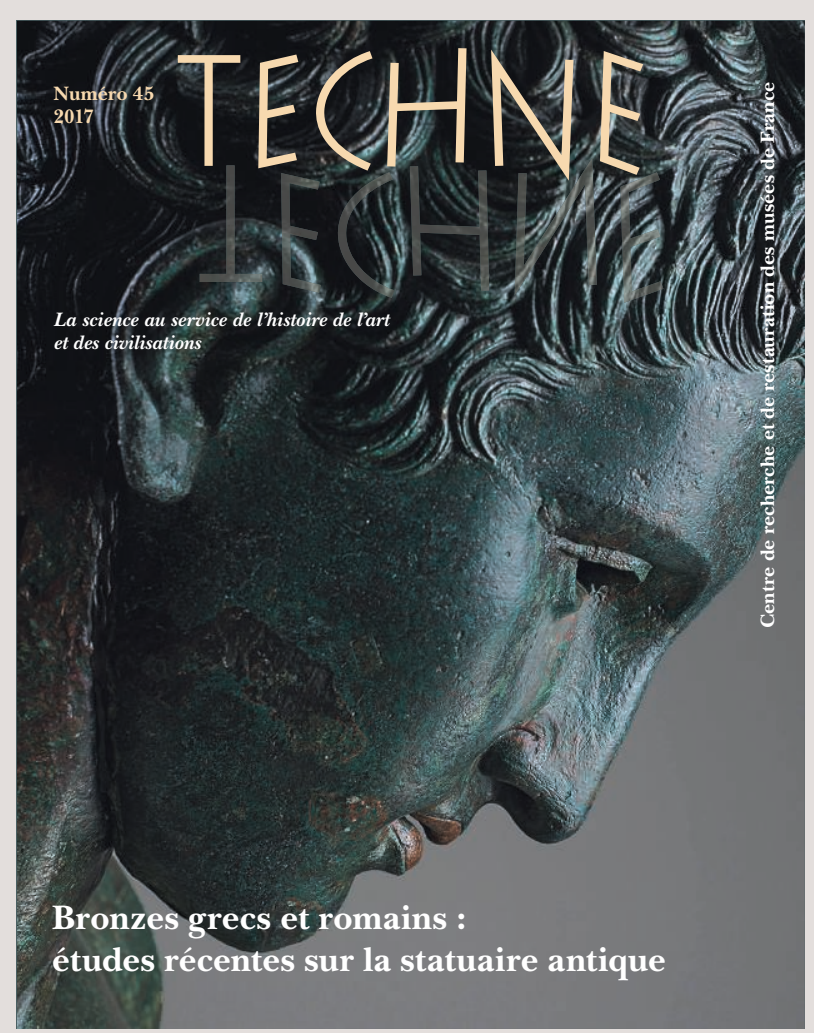

l'Apoxyomène de Croatie. En 2015 et 2017, dans le cadre des $19^{\mathrm{e}}$ et $20^{\mathrm{e}}$ colloques internationaux sur les bronzes antiques, ce sont la virtuosité de la production rhodienne à la fin du II $^{\mathrm{e}}$ siècle avant J.-C., illustrée par l'Apollon de Piombino, et les premiers résultats d'une nouvelle auscultation de l'Aurige de Delphes qui ont été abordés. Les travaux sur cette œuvre majeure se poursuivent actuellement sur la base d'un partenariat entre le musée du Louvre, le C2RMF, l'Éphorie des Antiquités de Phocide et l'École française d'Athènes. Ils déboucheront sur une journée d'étude à l'automne 2020 et sur une publication monographique en 2021. 
d'une grande difficulté, sont utiles, voire nécessaires, et représentent un travail d'expérimentation très louable - mentionnons notamment les propositions récentes de V. Brinkmann et U. Koch-Brinkmann dans ce domaine ${ }^{1}-$ mais elles n'ont de sens qu'établies avec la plus grande prudence, en s'appuyant exclusivement sur des résultats assurés. Il importe de faire la part entre données objectives, fondées sur les études techniques et physico-chimiques, et données subjectives. Il faut surtout se garder de surinterpréter, et avertir l'observateur moderne des limites de l'exercice, inhérentes à toute démarche fondée en grande partie sur l'extrapolation.

\section{Une polychromie surtout métallique}

Quelques traces ténues indiquent que les bronzes pouvaient être peints ${ }^{2}$. On ne peut cependant dire à l'heure actuelle si ce phénomène, qui n'a été qu'exceptionnellement observé, était développé ou non et s'il concernait également la grande statuaire de bronze.

Les effets de polychromie recherchés par les bronziers reposaient essentiellement sur des techniques propres au travail du métal et non sur les pratiques picturales adoptées par les sculpteurs sur pierre et par les coroplathes. La palette des bronziers, liée à des incrustations et à des placages de métaux purs ou alliés, était donc limitée, de même que les choix d'ordre esthétique qui s'offraient à eux et différenciaient leurs créations des sculptures en marbre ou en calcaire et des figurines en terre cuite. La polychromie des grands bronzes antiques était ainsi relativement restreinte, avec des contrastes spécifiques imposés par les procédés de mise en œuvre.

Aux époques archaïque et classique, les compléments métalliques permettaient d'ajouter le rouge, le blanc et le jaune à la teinte d'un jaune plus ou moins rosé de l'alliage cuivreux de base. Le cuivre rouge, peu ou non allié, concernait les lèvres ${ }^{3}$, les pointes des seins ${ }^{4}$ et le sang ${ }^{5}$; l'argent était utilisé pour les dents et, comme le cuivre ou l'or, pour les parures de tête ou le décor des vêtements ${ }^{6}$. La couleur venait également d'éléments rapportés (armes, bijoux, ustensiles, attributs spécifiques) qui pouvaient être en or, en argent ou en cuivre ${ }^{7}$. À l'époque hellénistique, les incrustations de cuivre rouge servaient encore à évoquer le sang, avec cependant parfois une approche plus complexe, conjuguant réalisme et dimension temporelle, ce dont témoigne le Pugiliste des Thermes, une œuvre qui incarne d'autant plus la période qu'il est impossible de la dater avec précision ${ }^{8}$. Les incrustations de cuivre rouge concernent les lèvres, les pointes des seins et les blessures infligées par l'adversaire. Mais la présence du cuivre rouge sur l'avant-bras droit de l'athlète est la conséquence non de blessures mais de la projection du sang à la suite du mouvement brutal de la tête. La répartition du sang a été anticipée par l'artiste avant la coulée avec l'aménagement, dans le modèle intermédiaire en cire, de cavités destinées à recevoir le cuivre. Par ailleurs, une tuméfaction sous l'œil droit, rendue par une incrustation dans un alliage cuivreux différent, était d'une teinte qui tranchait avec celle du visage. Le Pugiliste des Thermes illustre la prise en compte par le sculpteur de la possibilité d'employer des alliages différents afin d'enrichir aussi par la couleur le discours intrinsèque de l'œuvre, avec un raffinement qui force l'admiration.

\section{Une attention particulière portée aux visages}

Les effets de polychromie des grands bronzes antiques étaient particulièrement concentrés sur les têtes. Les travaux pionniers d'Edilberto Formigli sur les guerriers de Riace, publiés au début des années 1980 et complétés par les études menées entre 2009 et 2011 à l'occasion de la troisième phase de restauration des deux œuvres ${ }^{9}$, ont révélé la virtuosité des procédés de mise en couleur des visages et souligné le soin apporté aux moindres détails. Les lèvres du guerrier A, entrouvertes sur des incisives en bronze plaquées d'argent, ont une texture qui paraît naturelle car le cuivre rouge n'a pas été poli après la coulée mais a conservé une surface poreuse. Et la moustache, qui dissimule en partie la lèvre supérieure, est d'une autre teinte, car réalisée dans un alliage cuivreux plus riche en étain. La bouche obtenue en fonte pleine était fixée dans le modèle intermédiaire en cire avant l'ajout de la moustache en cire et la coulée de la tête ${ }^{10}$. Cette prouesse technique illustre l'excellence de bronziers qui maîtrisaient remarquablement vers 460 avant J.-C. les techniques de coulée à la cire perdue, combinant fontes en creux indirecte et directe et fonte pleine, avec la possibilité de modifier la composition élémentaire des alliages cuivreux ${ }^{11}$.

\section{L'importance accordée aux yeux}

L'une des principales sources de couleur des grands bronzes antiques venait de leurs yeux ${ }^{12}$. Selon la tradition grecque, attestée dès la fin du viII ${ }^{\mathrm{e}}$ siècle avec la triade apollinienne de Dréros, puis au viI ${ }^{\mathrm{e}}$ siècle avec les protomés de griffon ${ }^{13}$, les yeux étaient rapportés, c'est-à-dire fabriqués indépendamment et non coulés directement avec la tête ${ }^{14}$. Ils ont souvent disparu car leur montage était fragile et leurs matériaux convoités par les pilleurs antiques ${ }^{15}$.

Le globe oculaire, enchâssé le plus souvent dans des tôles martelées et découpées à leur extrémité sous forme de languettes pour imiter les cils ${ }^{16}$, pouvait être en marbre, en calcaire, en albâtre, en ivoire ou en os. Les paupières étaient en cuivre ${ }^{17}$. Une couleur orangée est bien souvent utilisée pour souligner le contour des orbites des œuvres en marbre ${ }^{18}$. La matière du globe oculaire était évidée au centre pour accueillir l'iris et la pupille. Des yeux isolés permettent d'apprécier la finesse du travail ainsi que la diversité des coloris et des matières ${ }^{19}$. Quand on songe aux dimensions des caroncules par rapport à la taille de statues plus grandes que nature, on est frappé par l'importance accordée à la restitution des couleurs de l'œil humain dès l'époque du style 


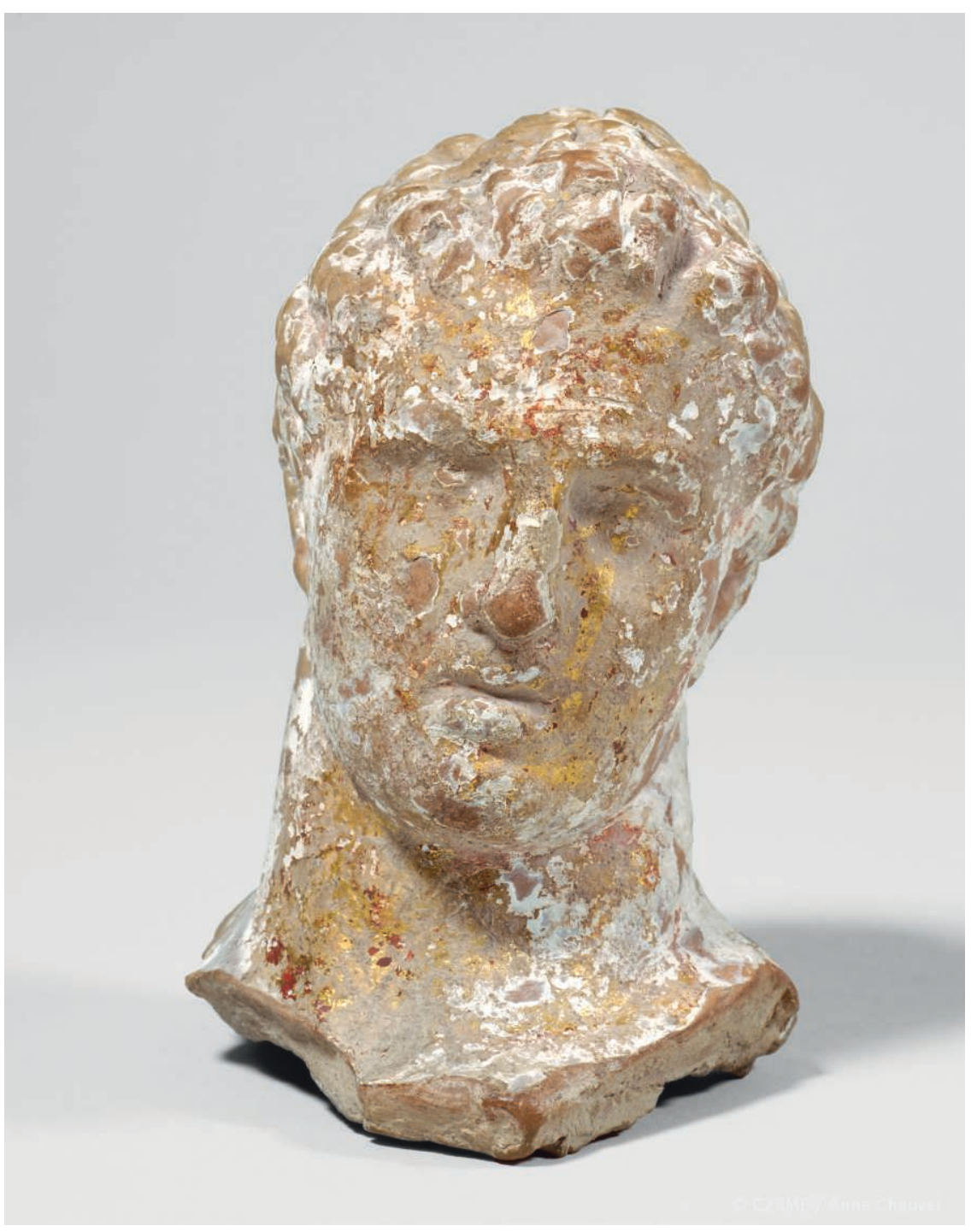

Fig. 2. Tête d'éphèbe, Smyrne, $\mathrm{II}^{\mathrm{e}}$ siècle av. J.-C.-II ${ }^{\mathrm{e}}$ siècle apr. J.-C., terre cuite dorée, Paris, musée du Louvre, département des Antiquités grecques, étrusques et romaines (CA 317). (C) C2RMF/A. Chauvet.

sévère ${ }^{20}$. Les yeux des bronzes grecs des $\mathrm{IV}^{\mathrm{e}}$ et III ${ }^{\mathrm{e}}$ siècles avant J.-C. gagnent en sophistication et se distinguent par un traitement caractéristique en verre coloré, comme en témoigne, vers 300 avant J.-C., le portrait en bronze de Seuthès III ${ }^{21}$.

\section{Couleur originelle des bronzes et témoignages iconographiques}

L'apparence actuelle des bronzes est trompeuse à cause des phénomènes de corrosion naturelle, liés à leur exposition durant l'Antiquité, aux traitements artificiels auxquels ils ont pu être soumis et aux conditions de leur enfouissement durant des siècles ${ }^{22}$. Leur teinte était à l'origine proche de celle d'un or jaune ou rosé, en fonction de la teneur plus ou moins élevée de l'étain dans l'alliage cuivreux. Les bronzes étaient raclés après la coulée à l'aide d'un outil métallique incurvé, afin d'éliminer la peau de fonte ${ }^{23}$, puis polis avec un os de seiche ${ }^{24}$ ou de la poudre d'émeri : les Anciens recherchaient l'éclat et la brillance du métal, qui variaient selon la nature de la lumière et la manière dont celle-ci se réfléchissait sur les surfaces lisses, incisées ou traitées plastiquement. Mentionnons, à titre d'exemple, les réponses nécessairement contrastées, pour un même éclairage, de la peau, de la barbe naissante et des mèches, épaisses et profondément nervurées, de la chevelure du souverain des Thermes ${ }^{25}$. Deux vases à figures rouges, respectivement de la fin du v $\mathrm{v}^{\mathrm{e}}$ siècle $^{26}$ et du premier quart $\mathrm{du} \mathrm{IV}^{\mathrm{e}}$ siècle avant J.-C. ${ }^{27}$, évoquent la couleur de base à laquelle aspiraient sculpteurs et commanditaires. Les corps métalliques y sont d'un jaune rehaussé de blanc. Sur le deuxième - un fragment de cratère en calice de Tarente -, la statue de culte d'Apollon a, pour la chevelure comme pour le corps, une même teinte jaune doré qui la distingue des motifs voisins réalisés selon la technique des figures rouges (fig. 1). Les traits du visage, les mèches de la chevelure et les contours des muscles sont notés avec un vernis délayé brun rouge. L'arc et la phiale tenus par le dieu sont rehaussés de blanc, pour imiter des attributs rapportés en argent. Dans le même temps, le dieu, figuré avec des cheveux sombres, est assis à l'extérieur de son temple. La différence entre le dieu représenté à l'instar d'un être vivant et son effigie de bronze est significative. Il est dès lors peu raisonnable de s'appuyer sur l'exemple antérieur de la coupe attique du Peintre de la Fonderie pour envisager l'existence, dès 490-480 avant J.-C., de patines intentionnelles noires sur les chevelures métalliques et pour les restituer dans les reproductions modernes ${ }^{28}$. Il ne faudrait toutefois pas en conclure qu'il n'y avait pas de patines dans l'Antiquité mais, d'après les indices matériels assurés relevés jusqu'à présent, cette pratique ne serait apparue que vers la fin de l'époque hellénistique.

\section{Des patines antiques, une conséquence de l'assombrissement naturel des bronzes?}

Les coroplathes de Smyrne ont produit, entre la fin de l'époque hellénistique et les premiers siècles de l'époque impériale romaine, des figurines qui s'inspirent de la statuaire 
Fig. 3. Héraclès, massif du Rhodope (Thrace) deuxième moitié du Iv ${ }^{\mathrm{e}}$ siècle av. J.-C., bronze doré, Paris, musée du Louvre, département des Antiquités grecques, étrusques et romaines (Br 487). (C) RMN-Grand Palais/Hervé Lewandowski.

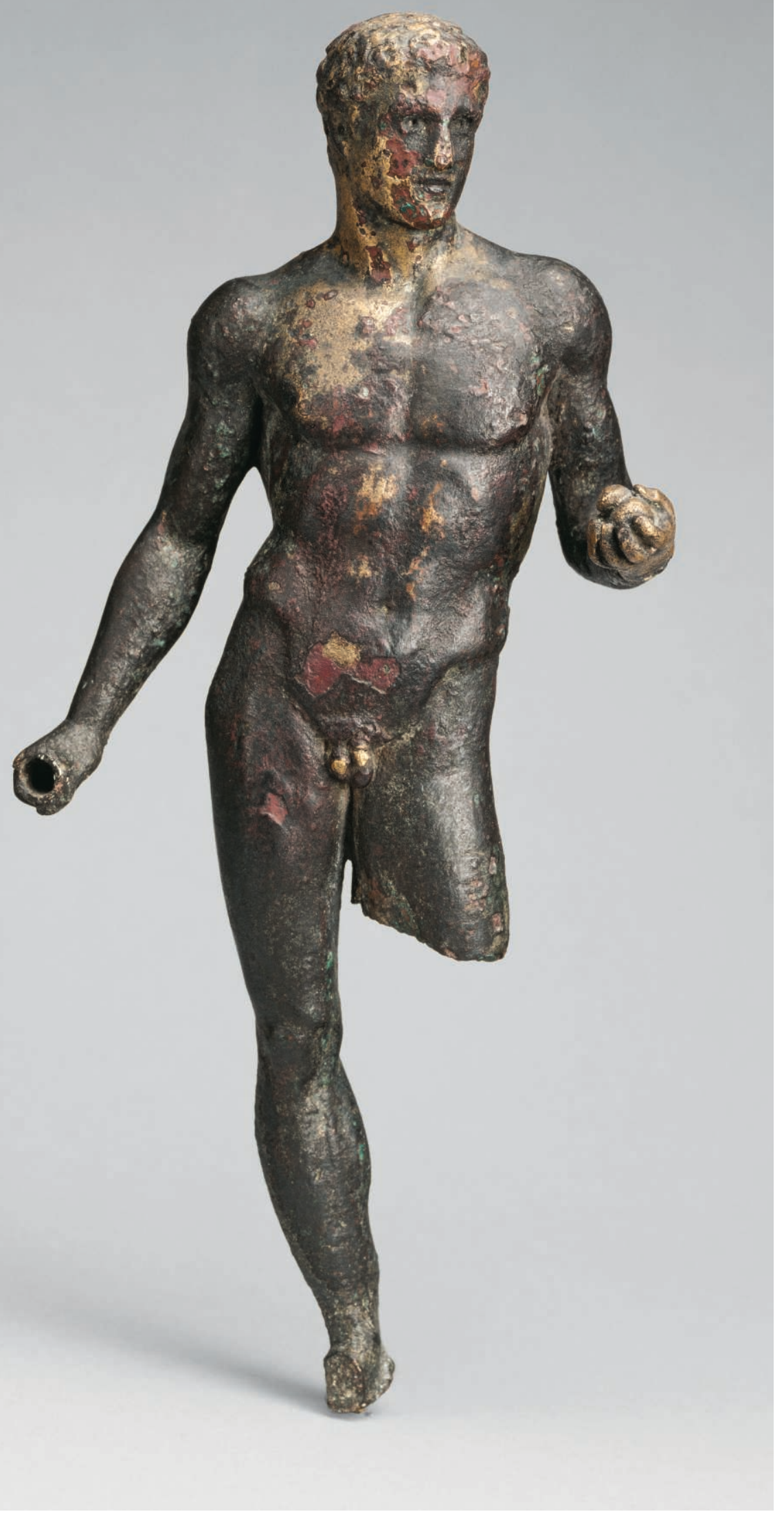




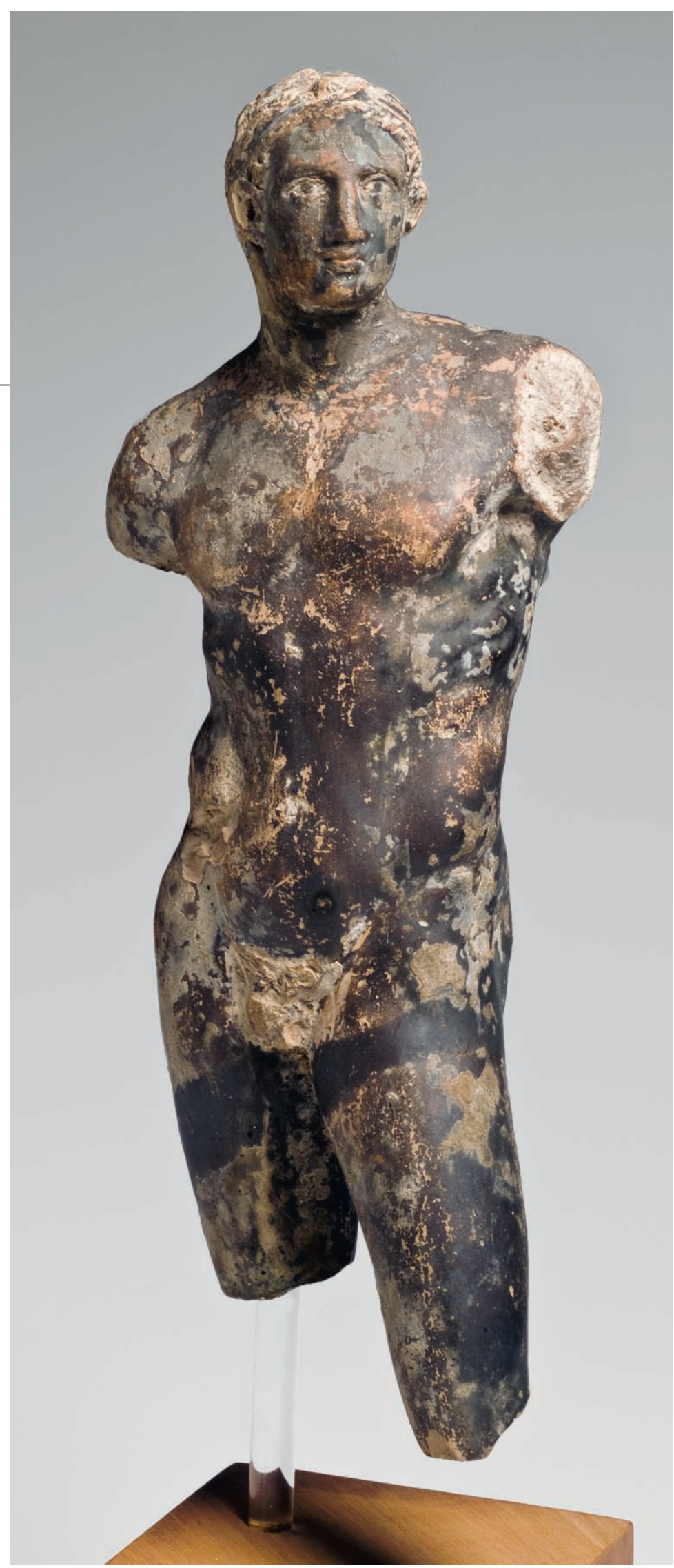

Fig. 4. Athlète ; Smyrne (?), $\mathrm{II}^{\mathrm{e}}-\mathrm{I}^{\mathrm{er}}$ siècle av. J.-C. (?), terre cuite peinte, Munich, Antikensammlung (inv. 6331). (C) Staatliche Antikensammlungen und Glyptothek München/R. Kühling. de bronze, grande et petite, des $\mathrm{v}^{\mathrm{e}}$ et $\mathrm{IV}^{\mathrm{e}}$ siècles av. J.-C., et nombreuses sont celles qui conservent encore sur leur épiderme quelques vestiges de feuilles d'or ${ }^{29}$. Elles confirment indirectement que les bronzes avaient une couleur proche de celle du métal précieux. On peut comparer une tête masculine imberbe en terre cuite et celle d'une grande statuette en bronze - un Héraclès qui tenait les pommes d'or du jardin des Hespérides - trouvée dans le massif du Rhodope $^{30}$ (fig. 2-3). L’une et l'autre sont les échos de créations lysippiques et toutes deux étaient entièrement dorées. La redondance métallique de l'Héraclès du Rhodope - l'or recouvre le bronze -, était la seule manière de garantir à l'œuvre une teinte pérenne. Mais le bronze étant instable et se corrodant vite sous l'effet des variations climatiques ${ }^{31}$, un tel parti esthétique, à savoir la couleur dorée des statues, imposait de tenter de ralentir le processus d'altération, c'està-dire de corrosion naturelle de l'alliage cuivreux. C'est le propos d'une inscription du début du III $^{\mathrm{e}}$ siècle avant J.-C., qui recommandait aux Agoranomes d'une cité de Grèce de l'Est d'entretenir une statue de tyrannoctone afin qu'elle ne s'altère pas et demeure brillante ${ }^{32}$. Cette évolution inéluctable était également la conséquence des traitements récurrents de protection des statues au fil des siècles. Les Anciens cherchaient en effet à retarder l'apparition de la corrosion, comme en témoignent quelques sources littéraires, en enduisant les épidermes de substances bitumineuses ${ }^{33}$. La couche de protection obtenue en délayant le bitume dans de l'huile d'olive pouvait être translucide et conserver au bronze son éclat ${ }^{34}$. Cependant, ces traitements, qui ne changeaient pas considérablement la teinte des bronzes au début, entraînaient paradoxalement un assombrissement progressif de l'alliage car de telles substances s'oxydent avec le temps ${ }^{35}$. Aussi l'évolution redoutée survenait-elle nécessairement assez vite et sans doute est-ce ce phénomène qu'il faut invoquer pour expliquer une figurine en terre cuite de Smyrne. Bien qu'imitant également un bronze, comme les autres terres cuites smyrniotes de ce type, elle est entièrement recouverte d'une couleur brune, à l'exception des yeux, aux sclérotiques blanches et iris sombres, et de la bouche légèrement ouverte sur une zone plus claire ${ }^{36}$ (fig. 4). Les restes de couverte noire sur le pied gauche du guerrier A de Riace et brune sur le cou de Seuthès doivent être interprétés comme la conséquence de traitements récurrents de protection par des substances contenant du soufre, et non comme des patines intentionnelles.

Il faut admettre que les œuvres des sculpteurs des $v^{e}$ et $\mathrm{IV}^{\mathrm{e}}$ siècles avant J.-C. n'avaient certainement déjà plus la même apparence quelques siècles après leur création. C'est cet état intermédiaire qui aurait modifié, dès l'Antiquité, le regard porté sur les bronzes et aurait entraîné un changement de goût, qui expliquerait peut-être l'apparition, avant la fin de l'époque hellénistique, de patines artificielles intentionnellement sombres ${ }^{37}$. 


\section{Vers une nouvelle esthétique des bronzes à la fin de l'époque hellénistique}

Une patine noire à base de soufre a été identifiée sur plusieurs bronzes de l'épave de Mahdia, antérieurs à la fin du II $^{\mathrm{e}}$ siècle avant J.-C. d'après la date du naufrage. Disposée de manière sélective, donc volontaire, cette couche d'un noir bleuté a pu être repérée sur un petit nombre de statuettes et d'éléments décoratifs : l'iris gauche de l'une des grandes statuettes de nain dansant est un clou, qui a été patiné avant son insertion dans le globe oculaire ${ }^{38}$. Il semble que l'épiderme du Jockey du cap Artémision et les sabots de sa monture présentent également les vestiges d'une patine noire antique ${ }^{39}$. Ce serait, pour la même époque, un autre témoignage des recherches nouvelles qui enrichissent alors la palette colorée des bronzes puisque cette œuvre peut être datée de manière plausible du milieu du $\mathrm{II}^{\mathrm{e}}$ siècle avant J.-C. ${ }^{40}$ Un enfant couronné de baies ou de petites perles, qui proviendrait du Fayoum et date du I ${ }^{\text {er }}$ siècle avant J.-C., a conservé presque intégralement sa patine sombre. Son corps d'un noir uniforme et comme lustré contraste avec ses yeux et ses dents en argent ${ }^{41}$. Une telle bichromie devient trichromie chez un autre enfant, peut-être Dionysos, qui ajoute à son corps noir et à ses yeux en argent le rouge cuivreux des rubans qui retombent de part et d'autre de son $\operatorname{cou}^{42}$. Le pilier hermaïque à tête de satyre d'une balustrade présente un même fond velouté noir sur lequel se détachent, avec les yeux en argent, les lèvres et la hampe verticale du thyrse qui sont en cuivre rouge ${ }^{43}$ (fig. 5). Ce sont très vraisemblablement ces « bronzes noirs » et leur polychromie singulière qui ont inspiré les sculpteurs romains lorsqu'ils ont choisi de travailler puis de polir des marbres noirs particulièrement sombres.

\section{«Bronzes de Corinthe » et jeux polychromes}

Une autre patine noire correspondait au moyen d'obtenir, grâce à des recettes qui ne sont pas précisément connues, le «bronze de Corinthe » des textes antiques ${ }^{44}$. Cet alliage fabuleux - il aurait été découvert fortuitement lors du sac de
Corinthe par les Romains en 146 avant J.-C., d'où son nom - ne se corrompait pas ${ }^{45}$. Il était susceptible de développer une patine pérenne d'un noir profond parce qu'il contenait de l'or et/ou de l'argent. En cela, il fascinait les Romains les plus riches et les alchimistes comme l'Égyptien Zosime de Panopolis, actif au III $^{\mathrm{e}}$ siècle de notre ère ${ }^{46}$. Les légendes rapportées ${ }^{47}$ insistent toutes sur l'association fortuite des trois métaux. Les premiers exemples attestés de «bronzes de Corinthe » existent bien mais datent au plus tôt du $\mathrm{I}^{\mathrm{er}}$ siècle de notre ère. Ce sont quelques rares œuvres tridimensionnelles, petites, composées de plusieurs parties fondues séparément dans des alliages différents, dont le "bronze de Corinthe ${ }^{48}$ ", et des incrustations. Ce ne sont jamais des grands bronzes. 
Quand il apparaît sous forme d'incrustations, l'alliage précieux est plus homogène car il a été martelé ${ }^{49}$. Et en effet, il ne se corrode pas avec le temps comme en témoigne un encrier de Vaison-la-Romaine sur la panse duquel six Érotes miment à Vénus, entourée de Psyché et d'une servante, la blessure fatale qui vient d'être infligée à Adonis. Le noir du cuivre patiné y est associé au rouge du cuivre pur, au blanc de l'argent et au jaune du laiton et de l'or ${ }^{50}$. Cette tétrachromie concernait également des éléments de mobilier et des sculptures mais toujours sous forme d'incrustations, ce que démontrent des fragments de cuirasse et de meubles d'Industria ${ }^{51}$ - possiblement un centre de production du « bronze de Corinthe » - et une grande statuette, possiblement galloromaine, figurant peut-être un Néron cuirassé ${ }^{52}$.

\section{Poikilletai}

Lorsqu'au deuxième quart du III $^{\mathrm{e}}$ siècle avant J.-C., Posidippe de Pella décrivait dans un poème ekphrastique le portrait en bronze de Philitas de Cos, conçu par Hécataios ${ }^{53}$, un verbe

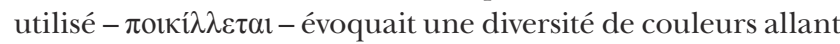
jusqu'à la bigarrure. On ne peut toutefois évaluer précisément le sens que l'auteur, qui maîtrisait manifestement parfaitement les procédés d'élaboration des bronzes, donnait à ce mot. Parlait-il de la palette métallique comme de l'éclat et de la brillance ? Vraisemblablement. L'introduction de la couleur noire, c'est-à-dire d'une première patine, garantie par les termini ante quos des cargaisons des bateaux naufragés, est, comme on l'a vu, postérieure de plus d'un siècle aux andriantopoiika de Posidippe. Jusqu'au $\mathrm{I}^{\mathrm{er}}$ siècle de notre ère, c'est la seule couleur, obtenue artificiellement, à être attestée dans le monde grec et romain. Une seule exception peut-être : la mensa isiaca découverte à Rome en 1527 et produite vers le $\mathrm{I}^{\text {er }}$ siècle dans un contexte isiaque, une grande table d'autel dont les divinités égyptiennes et les faux hiéroglyphes sont incrustés de métaux précieux et d'alliages patinés aux multiples nuances allant du brun, plus ou moins foncé, au noir velouté ${ }^{54}$. Par la suite, les patines se diversifient dans le monde romain, comme en témoigne, à Volubilis, le paludamentum damasquiné d'une statue colossale de Caracalla ${ }^{55}$. Cette multiplication des teintes est décrite par Zosime lorsqu'il énumère différentes recettes pour teinter le cuivre, notamment pour reproduire les carnations rouge sombre et beige rosé des figures masculines et féminines. On aimerait repérer ces nuances... Les phénomènes de corrosion qui affectent désormais les bronzes troublent le regard moderne, leur donnant des couleurs qu'ils n'avaient pas dans l'Antiquité.

\section{Notes}

1. Brinkmann, Koch-Brinkmann, $2018 \mathrm{a} ; 2018 \mathrm{~b}$

2. Un épisème de bouclier d'Olympie présente des restes de cinabre : Born, 1990, p. 188-192 ; id., 2004a, p. 126, 129-131, fig. 211, 213, 214 ; id., 2004b, p. 423 ; Philipp 2004, n 22 , p. 198-216, pl. 18-23.

3. Voir infra.

4. Les pointes des seins des statues masculines étaient coulées à part et rapportées. Formigli, 2013a, p. 285, fig. 366, p. 286.

5. Bol, $1978, n^{\circ} 416$, p. $64,92-93,134$ pl. 66. La pointe de lance fragmentaire d'une statue disparue d'Olympie, datée peutêtre de la première moitié $d u v^{e}$, est incrustée de bandes sinueuses de cuivre qui évoquent le ruissellement du sang.

6. Par exemple, le méandre de svastikas en cuivre sur la bordure de la chlamyde d'un cavalier du deuxième quart du $\mathrm{v}^{\mathrm{e}}$ siècle avant J.-C., Londres, British Museum, inv. GR 1886.3-24.2-6 : Williams, 1989, p. 534, 536538, fig. 11-12, 14-17 (de Tarente?). 7. Sur la polychromie des bronzes antiques, voir notamment Bol, 1985, p. 148157 ; Mattusch, 1996, p. 24-32 ; Wünsche, 2004, p. 135-137, 139-147 ; DescampsLequime, 2006 ; Formigli, 2013a et b ; Descamps-Lequime, 2015.

8. Rome, Museo Nazionale Romano di Palazzo Massimo, inv. 1055. Cat. Exp. Bonn, 1989 , n 1 , p. 150-180, 201-203, fig. 1a-e ; Formigli, 2013a, p. 274, 286-288, fig. 367368 ; Cat. Exp. Florence-Los AngelesWashington, 2015, p. 32, 222-223, n ${ }^{\circ} 18$ [J. M. Daehner].

9. Formigli, 1984 ; Donati, 2013, p. 273, n. 1.

10. Formigli, 1984, p. 130, 132, fig. 30 ; Donati, 2013, p. 268, p. 270-271, fig. 309 ; Formigli, 2013a, p. 282-283, fig. 353-356. À l'époque romaine, les lèvres de cuivre ne sont plus massives mais le plus souvent seulement plaquées.

11. Mattusch, 1988, p. 158 ; Mille, 2017, p. 298-300.

12. Formigli, 2013a, p. 275-280, particulièrement fig. 317-318.

13. Sphyrélata de la fin du viII ${ }^{\mathrm{e}}$ siècle avant J.-C. : la triade apollinienne de Dréros, Rolley, 1994, p. 112-113, fig. 98. Sur les protomés de griffon, voir l'exemplaire martelé d'Olympie, daté de 660-650 av. J.-C., qui a conservé ses yeux : Mallwitz, Herrmann, 1980, n 32, p. 64-65, pl. 32. 14. Bol, 1978 , p. $93-98$, pl. $68-70$; Lahusen, Formigli, 2001, p. 462-464, 466470 ; Formigli, 2013a, p. 276-277, fig. 319-328, 279, 280, fig. 344. À l'exception de quelques portraits étrusco-italiques et romains, dont les yeux, plaqués d'un matériau blanc, étaient coulés avec la tête - seul l'iris pouvant être rapporté - cette tradition se maintint 
jusqu'à l'époque hadrianique.

15. Formigli, 2013a, p. 278.

16. Formigli, 2013a, p. 278-279.

17. Sur le bord de tôle réinséré sous la paupière supérieure droite du guerrier $B$ de Riace, voir Donati, 2013, p. 270-271, fig. 310

18. Combattant perse du sarcophage dit d'Alexandre, Sidon, vers 320 av. J.-C., Istanbul, Musée archéologique, inv. 370 (Brinkmann, Koch-Brinkmann, 2010, p. 131, fig. 83).

19. Bol, $1978, n^{\circ} 422$, p. 95,135 , pl. 70 ; Formigli, 2013a, p. 278, 280, fig. 338 ; Donati, 2013, p. 270.

20. Petite pierre rosâtre au coin de l'œil droit du guerrier A de Riace : Donati, 2013 , p. 269-270, fig. 305 ; Formigli, 2013a, p. 278 , 280, fig. 339 .

21. Provenant de Shipka, près de Kazanlak en Bulgarie : Saladino, 2012-2013, p. 125-130, 169, fig. 1-4, 32b ; Formigli, 2012 2013, p. 184-185, fig. 43-48 ; Formigli 2013a, p. $279-281$, fig. 346 , 282 , fig. $347-350$.

22. Voir la reconstitution moderne en bronze du type dit de l'Apollon de Cassel, Rolley, 1994, p. 13, fig. 9

23. Coupe attique à figures rouges du Peintre de la Fonderie, face B, Berlin, Staatliche Museen, Antikensammlung, inv. F 2294.

24. Amphore attique à figures rouges, Peintre de Dutuit, vers 480 av. J.-C., Boston, Museum of Fine Arts, inv. 13.188.

25. Rome, Museo Nazionale Romano di Palazzo Massimo, inv. 1049. Cat. Exp. Bonn, 1989, n 4, p. 126-149, 205-207, fig. 4a-f, particulièrement p. 145-146.

26. Cratère à volutes attique à figures rouges, Ruvo, Museo Archeologico Nazionale G. Jatta, inv. 36933 (ex. 1501) ; DescampsLequime, 2015, p. 152-153, fig. 10.2.

27. Amsterdam, Allard Pierson Museum, inv. 2579, Mattusch, 1996, pl. 2.

28. Sur la coupe du Peintre de la Fonderie (supran. 23), les chevelures des statues de bronze sont traitées en vernis noir comme celles des figures humaines.

29. Cat. Exp. Paris, 2009, cat. 52, p. 117 Bourgeois, Jeammet, Pagès-Camagna, 20122013, p. 496-499, 501, 506-507, fig. 5-6, 8.

30. Paris, musée du Louvre, département des Antiquités grecques, étrusques et romaines, inv. CA 317 pour la terre cuite et $\mathrm{Br} 487$ pour le bronze.

31. Formigli, 2013b, p. 289.

32. Erythrée, Chios ou Clazomènes.

Zimmer, 1990, p. 153, n. 573

33. Pline, NH XXXIV, 99 ; Paus. I, 15, 4.

34. Wünsche, 2004, p. 142.

35. Formigli, 2013b, p. 291.

36. C'est Violaine Jeammet qui m'a signalé cette statuette de Smyrne, dont le traitement atypique peut être mis en relation avec l'assombrissement des bronzes et, probablement, l'existence de patines intentionnelles antiques. Je l'en remercie très sincèrement. Descamps-Lequime, 2006, p. 84-85 ; Bourgeois, Jeammet, PagèsCamagna, 2012-2013, p. 507-508, fig. 11.

37. Heilmeyer, 1994, p. 801-802 ; Born, 2004a, p. 128-129, fig. 212 : une fresque de Pompéi figure une scène de banquet avec une grande statuette patinée noire, posée sur une plinthe circulaire ; Formigli, 2013b, p. 289, 290-291, fig. 372

38. Willer, 1994, p. 1023-1026, fig. 1 et pl. 35.3. L'analyse de la patine du clou correspondant à l'iris gauche de la statuette de nain F 215 (Eggert, 1994, p. 1034) donne la composition suivante : $67 \%$ de cuivre, $32 \%$ de soufre.

39. Cette patine est particulièrement bien conservée sur le sabot antérieur gauche. Hemingway, 2004, p. 72, pl. 3, 5-10.

40. Hemingway, 2004, p. 92-99, 146-148.

41. The Saint Louis Art Museum, inv. 36:26, Cat. Exp. Cambridge, 1996, n 25 , p. $55,63,237-240,242$, pl. $4,14$.

42. The J. Paul Getty Museum, inv. 96.AB.53, Cat. Exp. Cambridge, 1996, n 26 , p. 237-242 ; Getty handbook, 2002, p. 181.

43. Paris, musée du Louvre,

département des Antiquités grecques, étrusques et romaines, inv. $\mathrm{Br}$ 4648.1-2

(Dossier C2RMF FZ 30488 ; Aucouturier $e$ al., 2010, p. 314-316, 320).

44. Pline, $N H$, XXXIV, 1 ; Plut., Mor.

395B-C; Giumlia-Mair, Craddock, 1993.

45. Cic., Tusc., IV, 14, 32

46. Giumlia-Mair, 2002 ; Hunter, 2002 ; Descamps-Lequime, 2005 ; 2006, p. 86-92 ; 2015, p. $97-98$; à paraître.

47. Pline, NH, IX, 139 ; Plut., Mor., VI,

395 C ; Florus, œuvres, I, 32.

48. Statuettes d'orateur noir d'Augst (Kauffmann-Heinimann, Liebel, 1994, $n^{\circ} 6$, p. 227-228, 231, fig. 1.6 ; Descamps-Lequime Robcis, 2019, p. 129-130, 133-134, fig. 2) et d'Avignon (C2RMF, FZ 36610, DescampsLequime, Robcis, 2019, p. 130-134, fig. 3-4, 6-8). p. 133

49. Descamps-Lequime, Robcis, 2019,

50. Descamps-Lequime, 2005, avec bibliographie antérieure ; Descamps-Lequime, Robcis, 2019, p. 133.

51. Mercando, Zanda, 1998, nos 60,61 , $65,67,73,74,197-199$, p. 58, 62, 112-116, 137 pl. D, I.2-3, L.1, LXIII-LXIV, LXVI, XCIIXCIII. Les incrustations en « bronze de Corinthe » sont identifiées alors à tort comme du nielle.

52. Cat. Exp. Anzio, 2009, p. 120-121

[L. Ceccarelli]. H. 55,9 cm.

53. Queyrel, 2010, p. 28-30, 33-37, 40 ;

Prioux, 2008, p. 144-145, 200 sq.

54. Turin, museo delle Antichità Egizie, inv. 7155, Cat. Exp. Londres, 2012, n 39, p. 139, 260 [E. Vassilika].

55. Boube-Piccot, 1969, p. 189-278 ;

Boube-Piccot, 2014, p. 35 ; Cat. Exp. Marseille, 2014, p. 114-117 [El Khayari] ; Aucouturier, Mathis, Robcis, 2017, p. 117-119, fig. 3-5.

\section{Bibliographie}

Aucouturier M., Mathis F., Robcis D., Castaing J., Salomon J., Pichon L., Delange E., Descamps S., 2010, "Intentional patina of metal archaeological artefacts: nondestructive investigation of Egyptian and Roman museum treasures", Corrosion Engineering, Science and
Technology, vol. 45, n 5, p. 314-321.

Aucouturier M., Mathis F., Robcis D., 2017, « Les bronzes noirs antiques - nouvelles observations et mécanismes de création ", dans S. Descamps-Lequime, B. Mille (dir.), Bronzes grecs et romains : études récentes sur la statuaire antique, Technè n ${ }^{\circ} 45$, p. 114-123.

Bol P. C., 1978, Grossplastik aus Bronze in Olympia (Olympische Forschungen 9), Walter de Gruyter, Berlin.

Bol P. C., 1985, Antike Bronzetechnik, Kunst und Handwerk antiker Erzbildner, Beck, München.

Born H., 1990, "Patinated and Painted Bronzes: Exotic Technique or Ancient Tradition?", dans M. True, J. Podany (dir.), Small Bronze Sculpture from the Ancient World. Symposium (Malibu, The J. Paul Getty Museum, 16-19 March 1989), The J. Paul Getty Museum, Malibu.

Born H., 2004a, « Zur Farbigkeit des Münchner Bronzekopfes mit der Siegerbinde ", dans Cat. Exp. Munich, 2004, p. 132-147.

Born H., 2004b, « Einlagen, Farbauftrag und Farbwirkung ", dans Philipp, 2004 p. 422-424.

Boube-Piccot C., 1969, Les bronzes antiques du Maroc. I. La statuaire (Études et travaux d'Archéologie marocaine IV), Rabat.

Boube-Piccot C., 2014, « Les bronzes antiques du Maroc », dans Cat. Exp. Marseille, 2014, p. 32-37.

Bourgeois B., Jeammet V., Pagès-Camagna S., 2012-2013, “'Color siderum'. La dorure des figurines en terre cuite grecques aux époques hellénistique et romaine », Bulletin de Correspondance Hellénique 136-137, p. 483-510.

Brinkmann V., Koch-Brinkmann U., 2010, "On the reconstruction of Antique Polychromy Techniques", dans V. Brinkmann, O. Primavesi, M. Hollein (dir.), Circumlitio. The Polychromy of Antique and Mediaeval Sculpture (Proceedings of the Johann David Passavant Colloquium, Frankfurt am Main, Liebieghaus Skulpturensammlung), Hirmer Verlag, Munich, p. 112-135.

Brinkmann V., Koch-Brinkmann U., 2018a "The Riace Bronzes Experiment. Aesthetics and Narrative", dans M. Fuchs (dir.), Ahoros. Gedenkschrift für Hugo Meyer von Weggefährten, Kollegen und Freunden, Phoibos, Vienne, p. 15-34

Brinkmann V., Koch-Brinkmann U., 2018b, « Die sogennanten Quirinalsbronzen und der Faustkampf von Amykos mit dem Argonauten Polydeukes. Ein archäologisches Experiment », dans Cat. Exp. Frankfurt am Main, 2018, p. 80-99.

Cat. Exp. Anzio, 2009, Anzio e Nerone. Tesori dal British Museum e dai Musei Capitolini [Exp. Anzio, 2009-2010], M. Sapelli Ragni (dir.), Ed. Gangemi, Rome.

Cat. Exp. Bonn, 1989, Herrscher und Athlet. Die Bronzen vom Quirinal [Exp. Bonn, Akademischen Kunstmuseum, 1989], N. Himmelmann (dir.), Olivetti, Milano.

Cat. Exp. Bonn, 1994, Das Wrack. Der antike 
Schiffsfund von Mahdia [Exp. Bonn,

Rheinisches Landesmuseum, 1994-1995],

G. Hellenkemper Salies et al. (dir.), Rheinisches Landesmuseum, Bonn.

Cat. Exp. Cambridge, 1996, The Fire of Hephaistos. Large Classical Bronzes from North American Collections [Exp. Cambridge, Toledo, Tampa, 19961997], C. C. Mattusch (dir.), Harvard University Art Museums, Cambridge-MA

Cat. Exp. Florence-Los Angeles-Washington, 2015, Power and Pathos. Bronze Sculpture of the Hellenistic World [Exp. Florence, Los Angeles, Washington D.C., 2015-2016], J. M. Daehner, K. Lapatin (dir.), Giunti Editore, Fondazione Palazzo Strozzi, J. Paul Getty Trust.

Cat. Exp. Frankfurt am Main, 2013, Zurück zur Klassik. Ein neuer Blick auf das alte Griechenland [Exp. Frankfurt am Main, 2013], V. Brinkmann (dir.), Hirmer Verlag, Munich.

Cat. Exp. Frankfurt am Main, 2018, Medeas Liebe und die Jagd nach dem goldenen Vlies [Exp. Liebieghaus

Skulpturensammlung, Frankfurt am Main, 2018-2019], V. Brinkmann (dir.), Hirmer Verlag, Munich.

Cat. Exp. Londres, 2012, Bronze [Exp. Royal Academy of Arts, Londres, 2012], D. Ekserdjian (dir.), Royal Academy of Arts, Londres.

Cat. Exp. Marseille, 2014, Splendeurs de Volubilis. Bronzes antiques du Maroc et de Méditerranée [Exp. Marseille, 2014], M. Morel-Deledalle (dir.), Actes Sud, Arles.

Cat. Exp. Munich, 2004, Bunte Götter. Die Farbigkeit Antiker Skulptur [Exp. Munich, 2004], V. Brinkmann, R. Wünsche (dir.), Staatliche Antikensammlung und Glyptothek, Munich.

Cat. Exp. Paris, 2009, D'Izmir à Smyrne. Découverte d'une cité antique [Exp. Paris, 2009], I. Hasselin-Rous, L. Laugier, J.-L. Martinez (dir.), Somogy Éditions/ musée du Louvre Éditions, Paris.

Cat. Exp. Paris, 2014, Moi, Auguste, empereur de Rome [Exp. Paris, Grand Palais, Rome, Galeries nationales, 2014], C. Giroire, D. Roger (dir.), RMN-Grand Palais Éditions, Paris.

Descamps-Lequime S., 2005, avec la collaboration de M. Aucouturier et de F. Mathis (C2RMF), "L'encrier de Vaisonla-Romaine et la patine volontaire des bronzes antiques ", Monuments et Mémoires de la Fondation Eugène Piot, t. $84 / 2$, p. $5-30$

Descamps-Lequime S., 2006, « La polychromie des bronzes grecs et romains ", dans A. Rouveret, S. Dubel, V. Naas (dir.), Couleurs et matières dans l'Antiquité. Textes, techniques et pratiques (Études de Littérature Ancienne 17), Éditions rue d'Ulm, Paris, p. 79-92.

Descamps-Lequime S., 2015, "The Color of Bronze: Polychromy and the Aesthetics of Bronze Surfaces", dans Cat. Exp. Florence-Los Angeles-Washington, 2015, p. 151-165.

Descamps-Lequime S. (à paraître), « Les bronzes de Corinthe: mythe ou réalité ?", Bulletin de la Société nationale des Antiquaires de France.
Descamps-Lequime S., Robcis D., 2019, “The Hellenistic Legacy of Metallic Polychromy: Roman Statuettes of an African Boy in the Pose of an Orator", dans S. Hemingway, K. Karoglou, Art of the Hellenistic Kingdoms. From Pergamon to Rome (The Metropolitan Museum of Art SYMPOSIA), The Metropolitan Museum of Art, New York, p. 127-135.

Donati P., 2013, « Die Gesichter der Bronzen von Riace. Technologische Aspekte, Untersuchungen und neue Ergebnisse ", dans Cat. Exp. Frankfurt am Main, 2013, p. 269-273.

Eggert G., 1994, « Schwarzfärbung oder Korrosion ? Das Rätsel der schwarzen Bronzen aus chemischer Sicht », dans Cat. Exp. Bonn, 1994, p. 1033-1039.

Formigli E., 1984, « La tecnica di costruzione delle statue di Riace ", dans L. Vlad Borelli, P. Pelagatti (dir.), Due Bronzi da Riace. Rinvenimento, restauro, analisi ed ipotesi di interpretazione (Bollettino d'Arte, 3, Serie speciale), Istituto poligrafico e zecca dello Stato, Roma, p. $107-142$.

Formigli E., 2012-2013, « Appendice : I danneggiamenti antichi al ritratto in bronzo di Seuthes III ", dans V. Saladino, 2012-2013, p. 180-186.

Formigli E., 2013a, « Die Oberflächengestaltung antiker Grossbronzen. Die Techniken der Farbigkeit ", dans Cat. Exp. Frankfurt am Main, 2013, p. 275-288.

Formigli E., 2013b, « Die "natürlichen” und künstlichen Patinae ", dans Cat. Exp. Frankfurt am Main, 2013, p. 289-292.

Getty handbook, 2002, The J. Paul Getty Museum handbook of the Antiquities Collection, The J. Paul Getty Museum, Los Angeles.

Giumlia-Mair A. R., 2002, "Zosime The Alchemist - Manuscript 6.29, Cambridge, Metallurgical interpretation", dans Giumlia-Mair A. R. (dir.), 2002, I Bronzi antichi: Produzione e tecnologia. Actes du colloque (GradoAquileia, 22-26 mai 2001), Monographies instrumentum 21, éditions Monique Mergoil, Montagnac, p. 317-323.

Giumlia-Mair A. R., Craddock P. T., 1993 , Corinthium Aes. Das schwarze Gold des Alchimisten, Philipp von Zabern, Mainz am Rhein.

Heilmeyer W.-D., 1994, « Zur Oberfläche antiker Grossbronzen », dans Cat. Exp. Bonn, 1994, p. 801-807.

Hemingway S., 2004, The Horse and Jockey from Artemision. A Bronze Equestrian Monument of the Hellenistic Period, University of California Press, Berkeley-Los AngelesLondon, 2004.

Hunter E., 2002, "Beautiful black bronzes: Zosimos' treatises in Cam. Mm.6.29", dans Giumlia-Mair A. R. (dir.), 2002, I Bronzi antichi : Produzione e tecnologia. Actes du colloque (Grado-Aquileia, 22-26 mai 2001), Monographies instrumentum 21, éditions Monique Mergoil, Montagnac, 2002, p. 655-660.

Kauffmann-Heinimann A., Liebel D., 1994, « Legierungen figürlicher Bronzen aus der Colonia Raurica ", Jahresberichte aus
Augst und Kaiseraugst 15, p. 225-237.

Lahusen G., Formigli E., 2001, Römische Bildnisse aus Bronze. Kunst und Technik, Hirmer, München.

Mallwitz A., Herrmann H.-V., 1980, Die Funde aus Olympia. Ergebnisse Hundertjähriger Ausgrabungstätigkeit, S. Kasas, Athènes.

Mattusch C. C., 1988, Greek Bronze Statuary. From the Beginnings through the Fifth Century BC, Cornell University Press, Ithaca and London.

Mattusch C. C., 1996, Classical Bronzes. The Art and Craft of Greek and Roman Statuary, Cornell University Press, IthacaLondon.

Mercando L., Zanda E., 1998, Bronzi da Industria, De Luca, Rome.

Mille B., 2017, D'une amulette en cuivre aux grandes statues de bronze : évolution des techniques de fonte à la cire perdue, de l'Indus à la Méditerranée, du $5^{e}$ millénaire au $5^{e}$ siècle av. J.-C., thèse en préhistoire, protohistoire et géosciences menée sous la direction de Catherine Perlès et de Vincent Serneels, université de Nanterre, université de Fribourg.

Philipp H., 2004, Archaïsche Silhouettenbleche und Schildzeichen in Olympia (Olympische Forschungen 30), Walter de Gruyter, Berlin-New York.

Prioux E., 2008, Petits musées en vers. Épigramme et discours sur les collections antiques, (Art et essai 5), Comité des Travaux Historiques et Scientifiques Institut National d'Histoire de l'Art, Paris.

Queyrel F., 2010, « Ekphrasis et perception alexandrine : la réception des œuvres d'art à Alexandrie sous les premiers Lagides ", Antike Kunst 53, p. 23-48.

Rolley C., 1994, La sculpture grecque 1. Des origines au milieu du $V^{e}$ siècle, Picard, Paris.

Saladino V., 2012-2013, « Il rittrato di Seuthes III », Jahrbuch des Deutschen Archäologischen Instituts 127/128, p. 125206.

Willer F., 1994, « Fragen zur intentionellen Schwarzpatina an den Mahdiabronzen ", dans Cat. Exp. Bonn, 1994, p. 10231031.

Williams D., 1989, "Knight rider, the Piot Bronze", Archäologischer Anzeiger, p. 529551.

Wünsche R., 2004, « Zur Farbigkeit des Münchner Bronzekopfes mit der Siegerbinde ", dans Cat. Exp. Munich, 2004, p. 132-147.

Zimmer G., 1990, Griechiche Bronzegusswerkstätten, Zur Technolgieentwicklung eines antiken Kunsthandwerkes, Philipp von Zabern, Mainz am Rhein, 1990. 